\title{
Surface Profiling of Object Using Varifocal Lens with Image Contrast
}

\author{
Chih-Yen Chen ${ }^{1}$, Lijuan Wang ${ }^{2}$, Chen-Liang Fan ${ }^{3}$, Pi-Ying Cheng ${ }^{3}$, Chun-Jen Weng ${ }^{1 *}$ \\ Instrument Technology Research Center, National Applied Research Laboratories, Taiwan \\ University of Kent, U.K. \\ Department of Mechanical Engineering, National Chiao Tung University, Taiwan
}

Phone: +886-3-5779911

Fax: +886-3-5773947

Email: cjweng@itrc.narl.org.tw

URL: http://www.itrc.narl.org.tw

\begin{abstract}
:
The measurement of automated and fast focusing is important in industrial inspection, biomedical microscopies. However, there are studies to survey the issues. This paper presents an imaging system with varifocal lenses is developed to obtain 3D surface topography of target samples. Experimental tests are conducted to evaluate the performance of the proposed optical imaging system. Experimental results demonstrate the feasibility and effectiveness of the proposed system. It is capable of constructing the multi-focus fusion with large depth of field and the 3D surface profile in almost real-time scanning.
\end{abstract}

Keywords: Varifocal lens; Autofocusing algorithms; Surface topography; Sharpness; Displacement measurement;

\section{Introduction}

Surface topography construction of an object in various depths is critical and challenging in an automatic imaging system. In order to realize automatic focusing, it is usually to look for the in-focus 
plane containing the highest sharpness along the z-axis of the specimen. However, sometimes the captured image only shows a focused image block with clear boundary while other image blocks are blurred. In this case, a variety of automated focusing techniques based on optical lens have been developed, such as distance measurement, non-contact characterization of samples, reconstruction of 3D surface topography measurement, etc [1-5]. In conventional imaging systems, optical lens with long focal length usually have a limited depth of field. Moreover, the focal length can be changed by mechanical translation using motors, slides and gears, to vary the relative distance between the lens and the sample [6,7]. In addition, high cost and complex operation of mechanical actuators have limited the applications of such systems.

In recent years, varifocal lenses have been reported and developed to implement the autofocusing without translation stage $[2,8]$. The basic principle of this method is to choose the sharper image blocks from the source images to construct a fused image or even the 3D surface topography, which contain a 'complete' measure of the scene than any of the individual source images $[10,11]$. The next procedure is to fuse complementary information from the selected images corresponding to individual focused objects [3]. The advantages of the imaging systems using varifocal lenses include compact system structure, fast response, easy-to-use and reliability [9]. However, there are very few studies describing the measurement of sharpness in the application of multi-focus image fusion. The measurement of image sharpness is highly dependent on the focus status, which is concerned to the autofocusing algorithms $[3,11,12,13]$.

In this study, an imaging system with varifocal lenses is developed to obtain 3D surface topography of target samples. In order to evaluate the performance of the proposed system, two different patterned substrates with wide stripes and narrow stripes, an aluminum block with a step-ladder pattern, and a metallic rod are used for the autofocusing evaluation. A total of twelve autofocusing algorithms including sum of absolute differences, sum of square differences, Brenner, Tenenbaum, Sum of modified Laplace, energy Laplace, Modified DCT, Diagonal Laplacian, Variance algorithm, Normalized Variance algorithm, Auto Correlation, Tenengrad variance are applied to obtain the textural details. Root Mean Square Error (RMSE) index is used to measure the similarity between any two images. 


\section{Experimental setup and analysis methods}

\subsection{Experimental setup}

As shown in Figure 1 the experimental setup consists of a microscope (Olympus BX51M), a CCD camera (EO-1312C-HQ LE), a varifocal lens (EL-16-40), and an objective lens (Olympus) . The varifocal lens is a shape-changing optical lens which is electrically tunable control. Thus, the focal length can be controlled arbitrarily by changing the curvature of the lens according to the current control. The driving current is within the range from $0 \mathrm{~mA}$ to $290 \mathrm{~mA}$ and provides the focal power from $-2 \mathrm{dpt}$ to $5 \mathrm{dpt}$. With the application of a 10X objective lens, the varifocal lens provides a maximum range wavelength of $8 \mathrm{~mm}$. This approach enables the rapid capture of sample images from a microscope operated at various focus distances. Switching time of the varifocal lens can be kept within less than $15 \mathrm{~ms}$, which means that the CCD camera system is capable of capturing hundreds of images within a few seconds. The objective lens is used to change the magnification and the resolution for the observation. When the lamp is turned on, the light travels through the varifocal lens and objective lens to the sample, from which it is directed to the CCD camera.

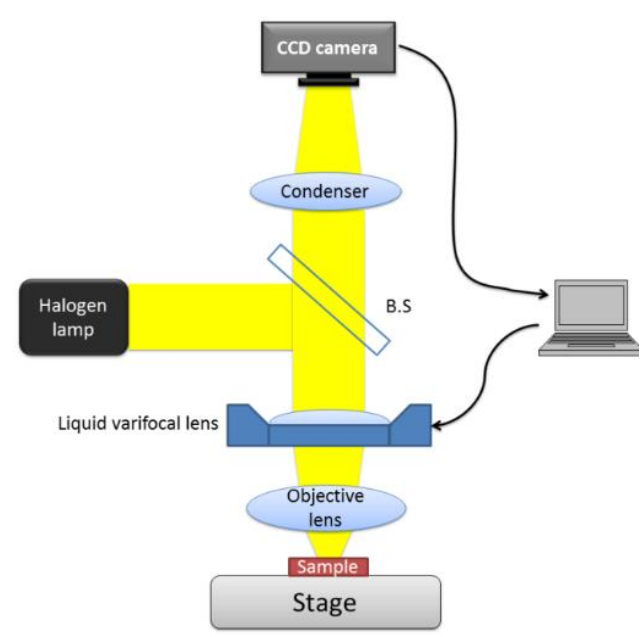

Fig. 1. Experiment setup by using varifocal lens for testing the various autofocusing algorithms.

Figure 2 is demonstraes the flowchart of the proposed system. 


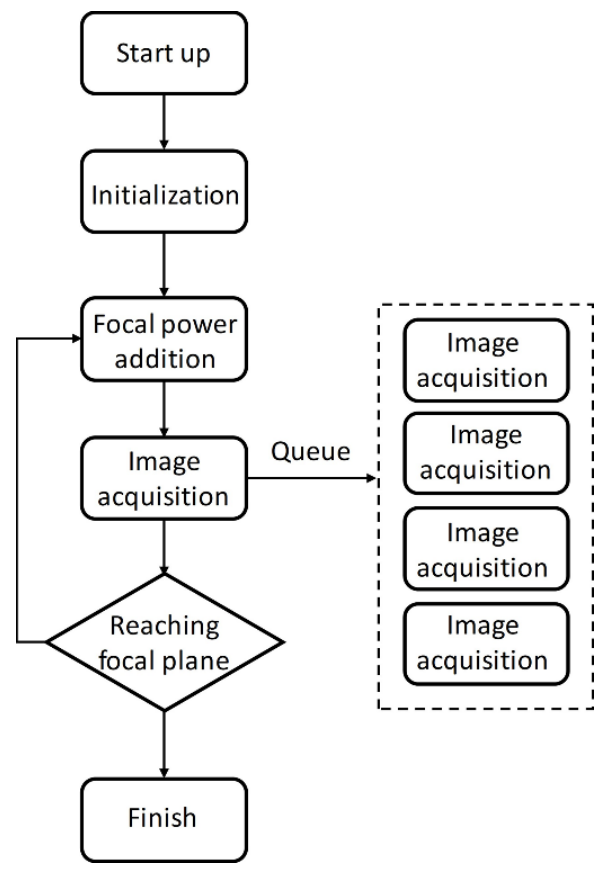

Fig. 2. The flowchart of the proposed system.

\subsection{Autofocusing algorithms:}

Twelve In order to evaluate the performance of the imageing system, different autofocusing algorithms were applieed and evaluated for each image obtained from the imaging system. There are twelve commonly used autofocusing algorithems and described as follows:

1) Sum of absolute differences $\left(F_{\text {abs_grad }}\right)$ :

$$
F_{\text {abs_grad }}=\sum_{\text {Height }} \sum_{\text {Width }}|I(x+1, y)-I(x, y)|
$$

The Height and Width are the height and the width of the obtained image, $I(x, y)$ respectively.

2) Sum of square differences $\left(F_{a b s \_g r a d}\right)$ :

$$
F_{\text {sq_arad }}=\sum_{\text {Height }} \sum_{\text {Width }}(I(x+1, y)-I(x, y))^{2}
$$

3) Brenner $\left(F_{\text {Brenner }}\right)$ :

$$
F_{\text {Brenner }}=\sum_{\text {Height }} \sum_{\text {Width }}(I(x+2, y)-I(x, y))^{2}
$$

4) Tenenbaum $\left(F_{\text {Tenen }}\right)$ :

$$
F_{\text {Tenen }}=\sum_{\text {Height }} I(x, y) \cdot S_{x}^{2}-I(x, y) \cdot S_{y}^{2}
$$


5) Sum of modified Laplace $\left(F_{S M L}\right)$ :

$$
F_{S M L}=\sum_{\text {Height }}\left|I(x, y) \cdot L_{x}\right|-\left|I(x, y) \cdot L_{y}\right|
$$

6) Energy Laplace $\left(F_{E L}\right)$ :

$$
F_{E L}=\sum_{\text {Height }} \sum_{\text {Width }}\left[I(x, y) \cdot L_{\text {energy }}\right]^{2}
$$

7) Modified DCT $\left(F_{M_{-} D C T}\right)$ :

$$
F_{M \_D C T}=\sum_{\text {Height Width }} \sum_{\text {Wid }} I \cdot M
$$

8) Diagonal Laplacian $\left(F_{D L}\right)$ :

$$
F_{D L}=\sum_{\text {Height }} \sum_{\text {Width }}\left|I(x, y) \cdot L_{x}\right|+\left|I(x, y) \cdot L_{y}\right|+\left|I(x, y) \cdot L_{d 1}\right|+\left|I(x, y) \cdot L_{d 2}\right|
$$
where $L_{d 1}$ and $L_{d 2}$

$$
L_{d 1}=\frac{1}{\sqrt{2}}\left[\begin{array}{ccc}
1 & 0 & 0 \\
0 & -2 & 0 \\
0 & 0 & 1
\end{array}\right] \quad L_{d 2}=\frac{1}{\sqrt{2}}\left[\begin{array}{ccc}
0 & 0 & 1 \\
0 & -2 & 0 \\
1 & 0 & 0
\end{array}\right]
$$

9) Variance algorithm $\left(F_{\text {variance }}\right)$ :

$$
F_{\text {variance }}=\frac{1}{H \cdot W} \sum_{\text {Height Width }} \sum_{\text {Wit }}(i(x+1, y)-\mu)^{2}
$$

where $\mu$ is the mean.

10) Normalized Variance algorithm $\left(F_{\text {nor_var }}\right)[12]$ :

$$
F_{n o r \_v a r}=\frac{1}{H \cdot W \cdot \mu} \sum_{\text {Height Width }} \sum_{\text {Wid }}(i(x+1, y)-\mu)^{2}
$$

11) Auto Correlation $\left(F_{\text {auto_corr }}\right)$ :

$$
\begin{aligned}
F_{\text {auto_corr }}= & \sum_{\text {Height }} \sum_{\text {Width }} i(x+1, y) \cdot i(x, y) \\
& -\sum_{\text {Height }} \sum_{\text {Width }} i(x+2, y) \cdot i(x, y)
\end{aligned}
$$

12) Tenengrad variance $\left(F_{T_{-} \text {variance }}\right)$ :

$$
F_{T \_ \text {variance }}=\left(T(x, y)-\mu_{T}\right)
$$

where $\mu_{T}$ is the mean of $T(x, y)$

$$
T(x, y)=\sqrt{I(x, y) \cdot S_{x}^{2}+I(x, y) \cdot S_{y}^{2}}
$$


where $D$ is the diopter index and $d_{n}$ is the focal power in layer $n$.

The maximum of the sharpness obtained from each algorithm is considered as the focus length $F(x, y)$ and compared with the reference R(x,y ). The RMSE (Root Mean Square Error) is used to quantify the difference between the calculated value and the reference and evaluate the performance of the focus measures.

$$
R M S E=\sqrt{\frac{\sum_{x} \sum_{y}[R(x, y)-F(x, y)]^{2}}{M \times N}}
$$

where $R$ and $F$ are reference image and fusion respectively, with size $M \times N$ pixels.

\section{Results and Discussion}

The herein reported novelty to address the autofocusing problem is not due to the development of just another focus measurement function but to explicitly apply the knowledge we had gathered to construct the 3-D profile measurement. To overcome the inherent disadvantages of the conventional autofocusing mechanisms, a new optical device together with a varifocal lens have been developed to build up a multiple in-focus imaging system. The advanced features in image acquisition are crucial for obtaining high-quality images even for scans over small regions. Therefore, two patterned substrates with wide stripes and narrow stripes, as shown in the Fig. 3(a) and (b), were used for testing the proposed 12 autofocusing algorithms. Based on the advanced features extracting from two patterned substrates, it could be benefit to the operation of autofocusing algorithms.
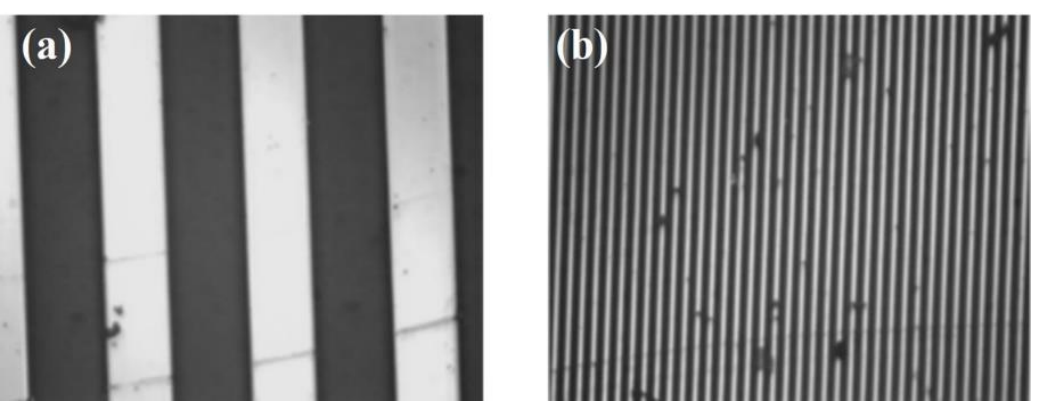

Fig. 3. Two patterned substrates with (a) wide stripes and (b) narrow stripes. 
Figure 4 shows the experimental results for wide stripes pattern and Figure 5 for narrow stripes pattern, which indicated the focus powers are presented from -3 to 3 , for narrow stripes. Besides, all the outputs which is showing the clarity of the should be normalized with respect to their maximum sharpness values. Results of output sharpness curves indicate that the patterned substrate with narrow stripes has shorter FWHM than with the wide stripes. This is because the narrow stripes contain more textural features and it is benefit to the detection of autofocusing algorithms.

(a)

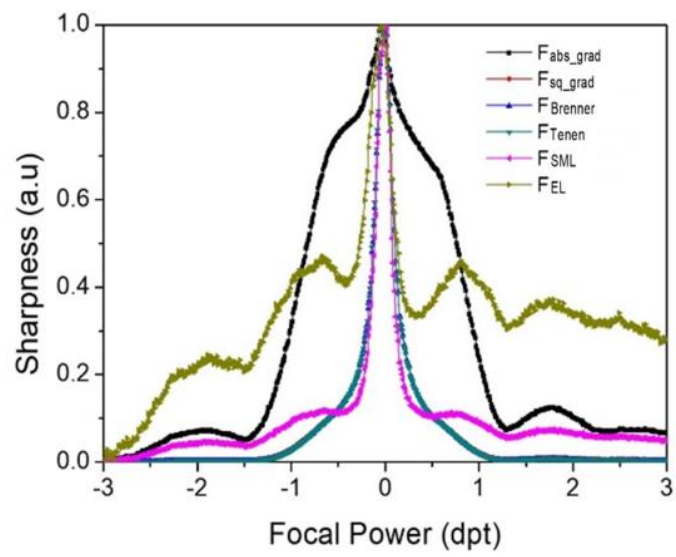

(b)

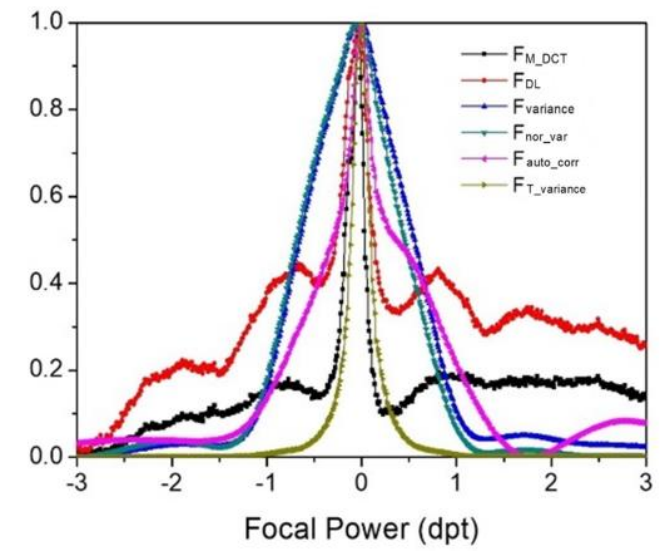

Fig. 4. Output sharpness curves for wide stripes from (a) autofocus algorithms 1 to 6 , and (b) autofocusing algorithms 7 to 12 .

(a)

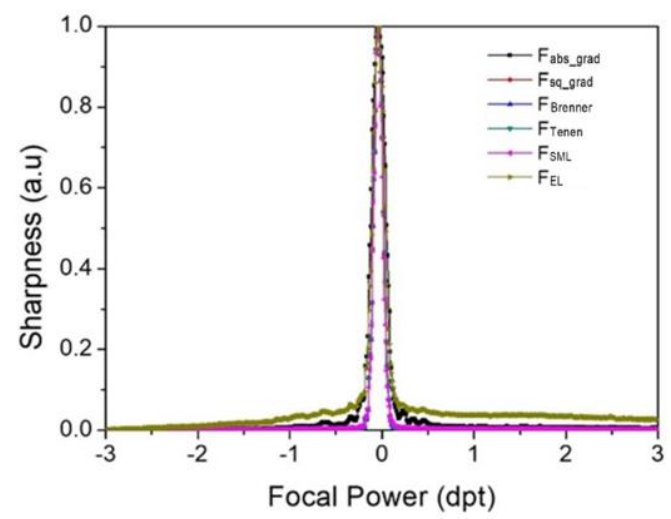

(b)

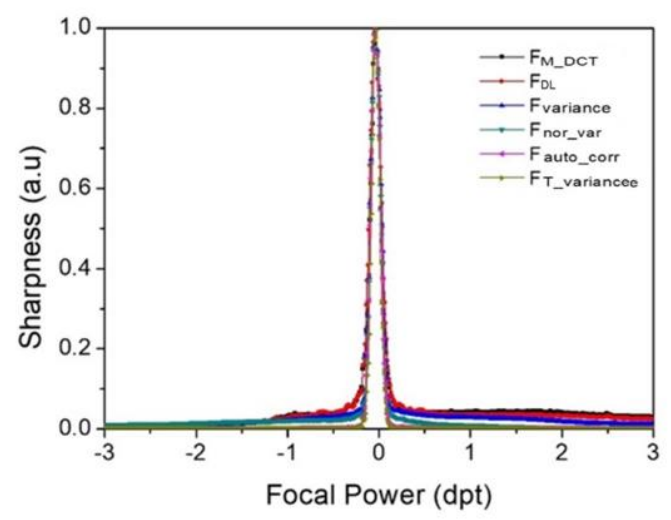

Fig. 5. Output sharpness curves for narrow stripes from (a) autofocusing algorithms 1 to 6 , and (b) autofocusing algorithms 7 to 12 . 
To verify the autofocusing algorithms, an aluminum block with a step-ladder pattern, as shown in Figure 6(a) was scanned across the light beam in a horizontal and lateral direction. The height of the aluminum block ranges from 50 to $100 \mu \mathrm{m}$. It is difficult to reconstruct an in-focus image due to the large difference in height. Figures 6(b) and (c) are the microscopic images from 10X objective lens with altering planes to fuse multiple in-focus depth images. Obviously, the limited depth-of-focus of optical lenses make it difficult to obtain an image with all relevant regions in focus.
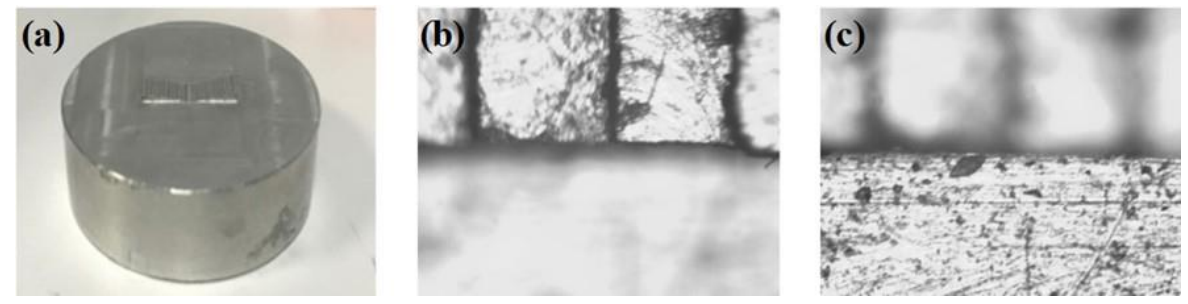

Fig. 6. Aluminum block with a step-ladder pattern was tested (a)Original image, (b)the focus on the ladder pattern and (c)the other plane.

In the following, the fusion of multiple in-focus depth images are reported. First, an initial position was manually set up in z-direction that lies within the upper and lower bounds of the test specimen. Subsequently, each image were taken at different focus depths to acquire the partially focused textural details. Hereby, the autofocusing algorithm, $F_{\text {abs_grad }}$, was applied with a fixed size of the search window, i.e. $w=28$. In order to find out the optimal in-focus planes for the 5 regions, a series of experiments was conducted. In this study, a total of 100 in-focus depth images were continually captured and a single stitched image preserving finest textures was saved. The resolution of microscopic images is $640 \times 512$, and the range of focal power is from $0.8 \mathrm{dpt}$ to $1.8 \mathrm{dpt}$. Figures 7 (a) and (b) presents the single stitched image of the aluminum block and the corresponding 5 measurements of the optimal focal power, respectively. 
(a)

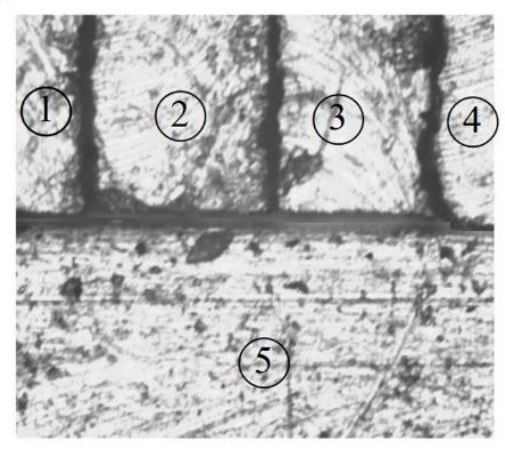

(b)

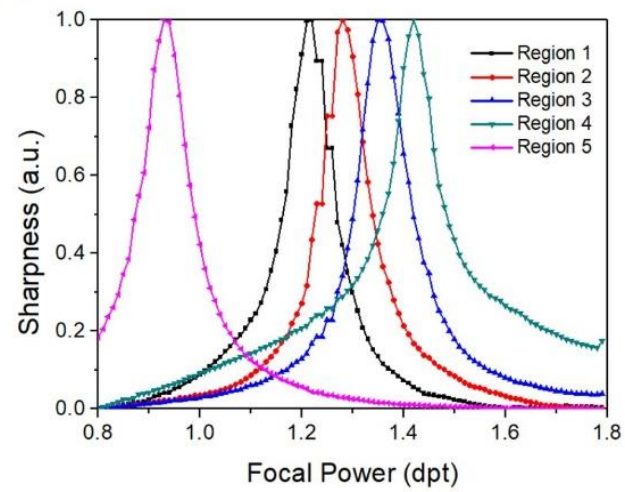

Fig. 7. (a) The single stitched image of the aluminum block and (b) the corresponding 5 measurements of the optimal focal power.

RMSE results with respect to parameter $w$ resulted from the 12 autofocusing algorithms are shown in Figure 8. Theoretically, the smaller RMSE indicates the better performance for the measured multiple in-focus images, i.e. the approaching of the in-focus plane. It can be observed that RMSE results of most autofocusing algorithms are decreasing dramatically when $w$ value is small. With an increase of $w$, the RMSE results of most autofocusing algorithms are slightly increasing. The only exception to this is the autofocusing algorithm of $F_{S M L}$. Additionally, the best RMSE result can be achieved by utilizing the $F_{\text {abs_grad }}$ with $w=25$. Windows size of $w$ is playing an improtant role in evaluating the autofocusing algorithms. So, how to choose a suitable parameter, $w$, for segmenting patterned textures to determine in-focus planes and depths is very critical. 
(a)

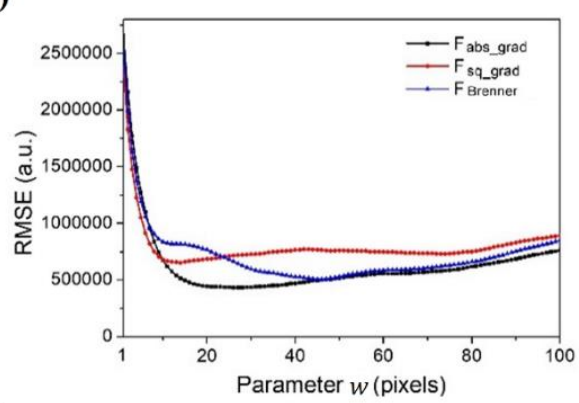

(c)

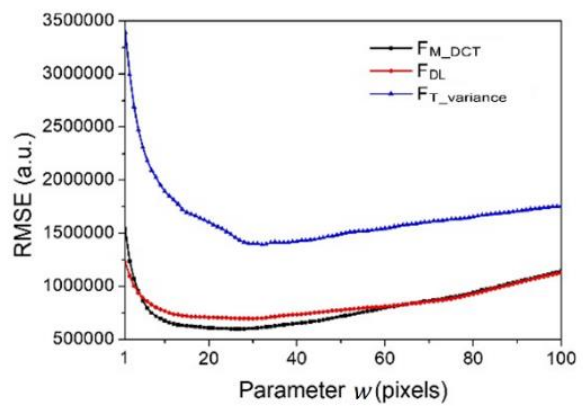

(b)

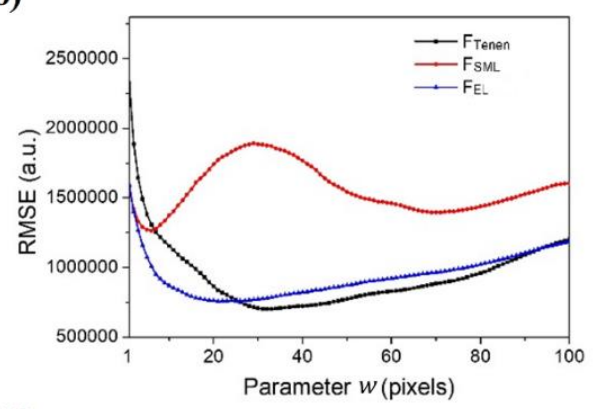

(d)

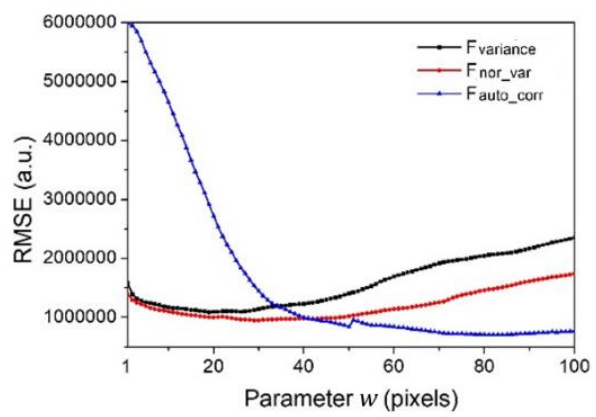

Fig. 8. RMSE results with respect to parameter $w$

\section{Curvature Measurement of the metallic rod}

This section presents the curvature measurement of the metallic cylinder which is based on the varifocal lens system. The autofocusing algorithm of $F_{\text {abs_grad }}$ with $w=28$ was used for the measurement in the range of 0 to $1 \mathrm{dpt}$. As shown in Figure 9(a) the metallic cylinder is in the diameter of $1.96 \mathrm{~mm}$. Figure 9(b) shows 3D profile of the curvature measurement and the comparison results between the experimental value and theoretical value are presented in Figure 9(c). Obviously, the measurement result of our proposed system has high agreement with the theoretical value.

(a)

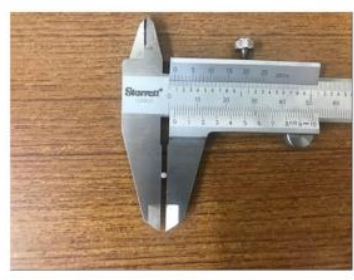

(b)

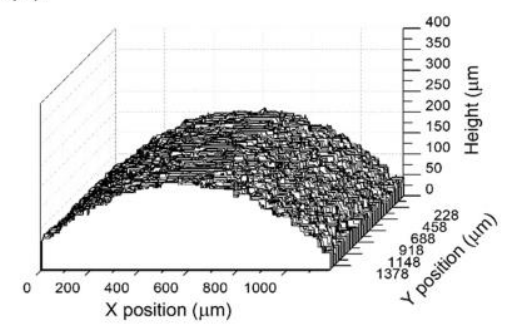

(c)

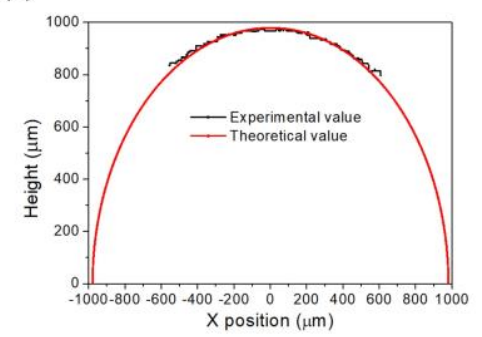

Fig. 9 Curvature measurement of the (a) metallic cylinder, (b) the surface profile and (c) the comparison between the experimental value and the theoretical value. 


\section{Conclusions}

This paper presents a novel imaging system with varifocal lenses. With the advantage of quick-reacting autofocus, the focal power of the imaging system can be operated in the range from $-3 \mathrm{dpt}$ to $3 \mathrm{dpt}$, and provide various resolutions arbitrarily. A total of twelve autofocusing algorithms have been applied to extract the sharpness of the images captured from the proposed system. To evaluate the performances of the autofocusing algorithms, experimental tests were conducted with two patterned substrates with wide stripes and narrow stripes, an aluminum block with a step-ladder pattern, and metallic cylinder, respectively, to detect the sharpness curves, surface profile of multi-focus fusion, and the curvature measurement. Experimental results demonstrate the feasibility and effectiveness of the proposed imaging system. It is capable of constructing the multi-focus fusion with large depth of field and the 3D surface profile in almost real-time scanning. Additionally, $F_{a b s \_g r a d}$ has demonstrated the best performance through experimental comparisons with other autofocusing algorithms. It is also proven that the $3 \mathrm{D}$ profile of multi-focus fusion can overcome the limited depth of field and provide a single stitched image with the corresponding 5 measurements of the optimal focal power.

\section{Acknowledgment}

This work was sponsored in part by the Ministry of Science and Technology, Taiwan, R.O.C. under contract numbers MOST 107-2221-E-492 -024 -MY3, 106-2622-E-492-004 -CC3 and MOST Add-on Grant for International Cooperation with

\section{References}

[1] Huang, W., \& Jing, Z. (2007). Evaluation of focus measures in multi-focus image fusion. Pattern recognition letters, 28(4), 493-500. 
[2] Casutt, S., Bueeler, M., Blum, M., \& Aschwanden, M. (2014, March). Fast and precise continuous focusing with focus tunable lenses. In Optical Components and Materials XI (Vol. 8982, p. 89820Y). International Society for Optics and Photonics.

[3] Wang, W., \& Chang, F. (2011). A Multi-focus Image Fusion Method Based on Laplacian Pyramid. JCP, 6(12), 2559-2566.

[4] C. J. Weng, B. R. Lu, P. Y. Cheng, C. H. Hwang, C.Y. Chen, "Measuring the thickness of transparent objects using a confocal displacement sensor," IEEE International Instrumentation and Measurement Technology Conference Proceedings, 978-1, 2017.

[5] Chen-Liang Fan, Chun-Jen Weng, Yu-Hsin Lin, Pi-Ying Cheng, "Surface profiling measurement using varifocal lens based on focus stacking," IEEE I2MTC 2018, pp. 1614-1618.

[6] Z. J. Ye, C. H. Hwang, P. Y. Cheng, C. Y. Chen, and C. J. Weng, "Rapid displacement sensor based on fitting scan,” IEEE International Instrumentation and Measurement Technology Conference Proceedings, 2016.

[7] C. J. Weng, T. H. Lan, C. H. Hwang, D. R. Liu, C. Y. Chen, P. Y. Cheng, and K. Y. Hsu, “Confocal displacement sensor with varifocal lens," IEEE International Instrumentation and Measurement Technology Conference Proceedings, 2015.

[8] Mateos-Pérez, J. M., Redondo, R., Nava, R., Valdiviezo, J. C., Cristóbal, G., Escalante-Ramírez, B., Jesús Ruiz-Serrano, M., Pascau J. \& Desco, M. (2012). Comparative evaluation of autofocus algorithms for a real-time system for automatic detection of Mycobacterium tuberculosis. Cytometry Part A, 81(3), 213-221.

[9] Blum, M., Bueeler, M., Graetzel, C. and Aschwanden, M., "Compact optical design solutions using focus tunable lenses," Proc. SPIE 8167, 81670W (2011).

[10] Li, S., Kwok, J. T., \& Wang, Y. (2001). Combination of images with diverse focuses using the spatial frequency. Information fusion, 2(3), 169-176.

[11] Li, S., Kwok, J. T., \& Wang, Y. (2002). Multifocus image fusion using artificial neural networks. Pattern Recognition Letters, 23(8), 985-997.

[12] Subbarao, M., Choi, T., Nikzad, A., 1992. Focusing Techniques. In: Proc. SPIE. Int. Soc. Opt. Eng., 163-174

[13] Nayar, S.K., Nakagawa, Y., 1994. Shape from focus. IEEE Trans. Pattern Anal. Mach. Intell. 16 (8), 824-831. 